\title{
BMO AND TEICHMÜLLER SPACE
}

\author{
D.H. Hamilton
}

\section{Introduction}

Let us consider the class $Q_{0}$ of quasiconformal maps $F$ of the Riemann sphere fixing $\infty$ and conformal at $\infty$ with normalization $f(z) \sim z$ at $\infty$. M. Reimann [18] proved that $\log \operatorname{det}(D F) \in \operatorname{BMO}\left(\mathbf{R}^{2}\right)$. We show the connection of this with a fundamental result of Teichmüller theory. Let $M_{0}$ be the class of $L^{\infty}$ functions $\mu$ with compact support and $\|\mu\|<1$. Ahlfors and Bers [3] show that the solution $F \in Q_{0}$ of the Beltrami equation

$$
\bar{\partial} F=\mu \partial F
$$

depends holomorphically on $\mu$. One formulation of Teichmüller space $T$ (see Becker [4], Gehring [11]) on a fixed domain $\Omega$ containing $\infty$ is to set

$$
T=\left\{\log F^{\prime}(z): F \in Q_{0} \text { conformal on } \Omega \text { with quasiconformal extension }\right\}
$$

Then it is proved that, if $\Omega$ is a quasidisk, $T$ is an open set in the space $\mathcal{B}(\Omega)$ of functions $g$ analytic on $\Omega$ with

$$
\|g\|_{\mathcal{B}}=\sup _{\Omega}\left|g^{\prime}(z)\right| \operatorname{dist}(z, \partial \Omega)<\infty, \quad|z g(z)| \rightarrow 0 \quad \text { as } \quad|z| \rightarrow \infty
$$

Now $\mathcal{B}(\Omega)$ is a closed subspace of the Bloch space. Coifman, Rochberg and Weiss [8] prove that $\mathcal{B}(\Omega)$ is the class of analytic functions BMO with respect to $\Omega$. Furthermore, by Jones [12], if $\Omega$ is a quasidisk every $g \in \mathcal{B}(\Omega)$ has an extension to a function of $\operatorname{BMO}\left(\mathbf{R}^{2}\right)$.

We remove the assumption that $\Omega$ is a quasidisk, and the restriction to considering $\log \partial F$ where $F$ is conformal. The formula $\log \partial F$ will be defined first for smooth $F$. Now we do not take the principle branch of $\arg \partial F$ but a continuous value so that $\log \partial F$ depends holomorphically on $\mu$ and is essentially unique.

Research supported in part by National Science Foundation Grant 8501509.

AMS classification: $30 \mathrm{C}$ 
Theorem 1. Suppose for $\mu \in M_{0}$ we let

$$
\Pi(\mu)=\log (\partial F),
$$

where $F=F^{\mu} \in Q_{0}$. Then the map $\Pi$ is a well defined holomorphic map of $M_{0}$ into $\mathrm{BMO}\left(R^{2}\right)$.

This has a number of implications.

Corollary 1. For any open set $\Omega$ with $\infty \in \mu$ and for any $F$ conformal on $\Omega$ with quasiconformal extension to $\mathbf{C}$ the function $\log F^{\prime}(z)(z \in \Omega)$ has an extension to $\mathrm{BMO}\left(\mathbf{R}^{2}\right)$.

We shall use the " $L^{2}$ norm" $\|\cdot\|_{*}$ for BMO, see Section 2.

Corollary 2. For any $\mu, \nu \in M_{0}$ with corresponding $F, G \in Q_{0}$

$$
\|\log \partial F-\log \partial G\|_{*} \leq K\|\mu-\nu\|_{\infty}+O(\|\mu-\nu\|)^{2}
$$

with $K=K(\|\nu\|)$. In particular at $G=z$

$$
\|\log \partial F\|_{*} \leq 3\|\mu\|_{\infty}+O\left(\|\mu\|_{\infty}\right)^{2},
$$

and 3 is best possible.

Remark. Reimann [19] obtained an upper bound for $\log \operatorname{det}(D F)$ which tends to 0 as $\|\mu\|_{\infty} \rightarrow 0$. The 3 comes from Iwaniec's bound for the Hilbert transform [13].

Let $\Omega$ be a domain containing $\infty$, and $M(\Omega)$ the subset $\mu \in M_{0}$ which are supported by $\mathbf{C} \backslash \Omega$. The map

$$
\Pi: M(\Omega) \rightarrow \log \left(F^{\prime}(z)\right)
$$

is a holomorphic map into $B(\Omega)$, see Ahlfors and Bers [3].

Bers [5] and Gehring [11] ask for a characterization of domains with a univalence criteria, i.e., domains for which there exists $a=a(\Omega)$ so that for any $g \in \mathcal{B}(\Omega)$ with $\|g\|_{\mathcal{B}}<a$ then there is $F$ conformal on $\Omega$ with $\log F^{\prime}=g$.

Now let $\mathcal{H}$ be the Hilbert transform

$$
\mathcal{H} f(z)=-\frac{1}{\pi} \int_{\mathbf{C}} \frac{f(\xi)}{(\xi-z)^{2}} d m(\xi) .
$$

A classical result of Fefferman and Stein [9] implies for $\delta \in L^{\infty}\left(\Omega^{C}\right)$ that $g=$ $\mathcal{H} \delta \in \operatorname{BMO}\left(\mathbf{R}^{2}\right)$ and thus $\left.g\right|_{\Omega} \in \mathcal{B}(\Omega)$. Bers [5] essentially proves the converse, i.e.,

$$
\mathcal{B}(\Omega)=\mathcal{H} L^{\infty}\left(\Omega^{C}\right),
$$


provided $\Omega$ is a quasicircle. We show that this integral representation for $\mathcal{B}(\Omega)$ (which is stronger than BMO extension property for elements in $\mathcal{B}(\Omega)$ ) holds if and only if $\Omega$ has a univalence criteria.

The equivalence occurs by the application of the $\lambda$-lemma of Sullivan, Thurston [22] and Bers, Royden [7] to the structure of the "pseudo" Teichmüller space

$$
S=\left\{\log F^{\prime}: F \text { conformal on } \Omega \text { and } F=z+O(1) \text { near } \infty\right\} .
$$

A complex curve $L \subset S$ is a holomorphic map $\Lambda:\{|\lambda|<1\} \rightarrow S$ with tangent $\tau$ at $\lambda=0$ given by $\tau=\left.(\partial \Lambda / \partial \lambda)\right|_{\lambda=0}$. The set of tangents at $g=\Lambda(0)$ is the tangent space $V_{g}$ at $g$. Clearly $V_{g}$ is a subset of $\mathcal{B}(\Omega)$ and if $0 \in \operatorname{Int} S$ then $V_{0}=\mathcal{B}(\Omega)$. We answer a question of Bers [6] who asked when $V_{0}=\mathcal{B}(\Omega)$. Shiga used [7], [22] to characterize $V_{g}$.

Theorem 2. The following are equivalent for an open set $\Omega$ with $\infty \in \Omega$ :

(i) $\Omega$ has a univalence criteria;

(ii) the tangent space $V_{0}=\mathcal{B}(\Omega)$;

(iii) $\mathcal{B}(\Omega)$ has integral representation, i.e.,

$$
g(z)=\mathcal{H} \delta, \quad \delta \in L^{\infty}\left(\Omega^{C}\right) .
$$

Remarks. 1) Kra [13] independently obtained similar results for Jordan domains.

2) It is interesting that the linear condition (iii) implies the nonlinear condition (i).

Finally we note that (iii) implies that (i) is actually a local condition.

Corollary 3. Let $\Omega$ be a domain so that $\Omega^{C}$ is the union of disjoint compact sets $A_{1}, A_{2}$. Then $\Omega$ has a univalence criteria if and only if $A_{1}^{C}, A_{2}^{C}$ do.

Instead of assuming $\mu$ has compact support we could just as well consider

$$
M=\left\{\mu \in L^{\infty}(\hat{\mathbf{C}}):\|\mu\|_{\infty}<1\right\}
$$

with normalization for solutions $F$,

$$
F(0)=0, \quad F(1)=1, \quad F(\infty)=\infty .
$$

It is not so clear how to define $\log (\partial F)$ even in the smooth case. Nevertheless we prove

Theorem 3. The map $\Pi: M \rightarrow \log (\partial F)$, for quasiconformal $F=F^{\mu}$ solving $\bar{\partial} F=\mu \partial F$ and fixing 0,1 and $\infty$, is a well defined holomorphic map of $M$ into $\operatorname{BMO}\left(\mathbf{R}^{2}\right)$. 
Finally we shall observe that this theory actually gives a new result for singular integrals.

Corollary 4. For any $\delta \in L^{\infty}$ (supported on a compact set)

$$
\mathcal{H}(\delta \mathcal{H} \delta)-\frac{1}{2}(\mathcal{H} \delta)^{2} \in \mathrm{BMO}\left(\mathbf{R}^{2}\right) .
$$

Remark. In general $(\mathcal{H} \delta)^{2}$ or $\mathcal{H}(\delta \mathcal{H} \delta)$ do not belong to $\operatorname{BMO}\left(\mathbf{R}^{2}\right)$.

This quadratic formulae in $\mu$ easily yields a commutator type result. For $\mu \in L^{\infty}$ define the operator $Q=\langle\mu, \mathcal{H}\rangle$ by

$$
Q f=\mathcal{H}(\mu \mathcal{H} f)+\mathcal{H}(f \mathcal{H} \mu)-(\mathcal{H} \mu)(\mathcal{H} f) .
$$

Corollary 4 proves that $Q$ is a bounded operator from $L^{\infty}$ to BMO. Coifman, Rochberg and Weiss [8] prove that the operator

$$
W f=\mathcal{H}(f \mathcal{H} \mu)-(\mathcal{H} \mu)(\mathcal{H} f)
$$

is a bounded operator of $L^{p}, 1<p<\infty$. Their result does not hold for $p=\infty$ so that the $Q$ operator is the correct generalization, note also that the first term of the $Q$ operator

$$
\mathcal{H}(\mu \mathcal{H} f)
$$

is a bounded operator on $L^{p}, 1<p<\infty$, for $\mu \in L^{\infty}$.

\section{Preliminary results}

In this section we collect some tools necessary for the proof of the main theorem. We shall be using $\operatorname{BMO}\left(\mathbf{R}^{2}\right)$ with " $L^{2}$ norm"

$$
\|f\|_{*}=\left(\sup _{Q} f_{Q}\left|f-f_{Q} f d x d y\right| d x d y\right)^{1 / 2}
$$

taken over all disks $Q$, where $f_{Q}$ is the mean value. The basic theory of BMO is given by Fefferman and Stein [9], where it is proved that BMO is the dual of the Hardy space $H^{1}$. In particular, the unit ball of BMO is weakly compact and since for $1<p<\infty$ the set

$$
E=\left\{u \in L^{p}\left(\mathbf{R}^{2}\right): \operatorname{supp} u \text { compact, } \int_{\mathbf{R}^{2}} u d m=0\right\}
$$

is dense in $H^{1}$, for each sequence $\left\{f_{n}\right\} \subset \mathrm{BMO}$ with $\left\|f_{n}\right\| \leq K^{1}$ there is a subsequence $\left\{f_{n}\right\}$ and an $f \in \mathrm{BMO}$ so that for any $h \in E$

\footnotetext{
$1 K$ denotes a constant which may vary from line to line, any dependence on parameters is made explicit, e.g.: $K=K(m)$ with $m=\|\mu\|_{\infty}$.
} 


$$
\int_{\mathbf{R}^{2}} h f_{n_{k}} d x d y \rightarrow \int_{\mathbf{R}^{2}} h f d x d y, \quad k \rightarrow \infty .
$$

A map $\chi: M_{0} \rightarrow$ BMO is holomorphic if for each $\mu_{0}$ and $\mu_{1}$ in $L^{\infty}$, with $\mu_{0}+\lambda \mu_{1} \in M_{0}$ for $|\lambda|<1$, the map $\chi\left(\mu_{0}+\lambda \mu_{1}\right)$ is holomorphic in $\lambda$. This is well known to be equivalent to the stronger condition that $\chi$ has continuous (Gateaux) differential.

Now Montel's theorem does not hold for Banach spaces but there is the following weak form. This is based on the fact that a map $\chi:\{|\lambda|<1\} \rightarrow$ BMO will be holomorphic if and only if $\int h(z) \chi(\lambda) d x d y$ is analytic for all $h \in E$. Thus given a sequence $\chi_{n}$ of holomorphic maps from $\{|\lambda|<1\}$ into BMO so that $\left\|\chi_{n}\right\|_{*} \leq K$ then there is a limit $\chi$ so that $\chi_{n} \rightarrow \chi$ weakly, i.e., for any $h \in E$ and compact $U \subset\{|\lambda|<1\}, \int h \chi_{n}(\lambda) d x d y \rightarrow \int h \chi(\lambda) d x d y$ uniformly for $\lambda \in U$.

The theory of quasiconformal solutions of

$$
\bar{\partial} F=\mu \partial F, \quad \mu \in M_{0},
$$

may be seen in Ahlfors and Bers [3]. Thus $F(z)=z+o(1)$ near $\infty$ and we have the canonical solution $F=F^{\mu}$ :

$$
F(z)=z+\mathcal{C} \mu+\mathcal{C} \mu \mathcal{H} \mu+\cdots,
$$

where $\mathcal{C}$ and $\mathcal{H}$ denote the Cauchy and Hilbert transforms. It is important that $F-z$ depends holomorphically on $\mu$ (in the above simple minded way) as a map into the space of bounded continuous functions. Now let $D F$ be the differential of $F$, then $\mu \in C_{0}^{\infty}$ implies $D F$ and $D F^{-1}$ are both $C^{\infty}$, see [16], p. 233. M. Reimann considered the function

$$
g(z)=\log \operatorname{det}|D F|
$$

and proved $g \in \mathrm{BMO}$ with norm only dependent on $\|\mu\|_{\infty}$. As a simple observation we have

Lemma 1. For canonical $F=F^{\mu}, \log |\partial F|$ is $\mathrm{BMO}$ and

$$
\|\log |\partial F|\|_{*} \leq K\left(\|\mu\|_{\infty}\right) .
$$

We have only to write

$$
\log \operatorname{det}|D F|=\log |\partial F|^{2}\left(1-|\mu|^{2}\right)=2 \log |\partial F|+\log (1-|\mu|)
$$

and observe

$$
\log \left(1-|\mu|^{2}\right) \in L^{\infty} \subset \text { BMO }
$$


We now consider the definition of $\log (\partial F)$. This is no problem when $\mu \in C_{0}^{\infty} \cap$ $M_{0}$ and may be accomplished in two different ways. One way is to take $\log (\partial F)=$ 0 at $\infty$ and use a path $\gamma$ from $\infty$ to $z$, along which $\log (\partial F)$ is continuously defined. It is clear that this $\log (\partial F)$ is uniquely defined independently of $\gamma$. A second way is to consider a continuous deformation of $\mu$ to 0 ,

$$
\mu(t)=t \mu, \quad 0 \leq t \leq 1,
$$

and an associated family $F^{t}$

$$
\bar{\partial} F^{t}=t \mu \partial F^{t}
$$

of canonical maps. Beginning with $F^{0} \equiv z$ and $\log \partial F=0$ we may continuously define $\log \partial F^{t}(z)$ at fixed $z$. It is clear that this definition agrees with the first (by the usual homotopy argument) and in fact any $C^{\infty}$ deformation of $\mu$ into 0 may be used. Thus $\log (\partial F)$ is uniquely defined for the $C^{\infty}$ case.

Now for the general case, as $F$ may be totally singular on a circle, the first method fails. It is necessary to show that the second method may be generalized and gives $\log \partial F$ uniquely, whatever the representation. Thus we begin with the $C^{\infty}$ case and establish BMO bounds. There are various difficulties caused by the fact the $C_{0}^{\infty} \cap M_{0}$ is not $L^{\infty}$ dense in $M_{0}$, and a type of weak convergence for sequences of quasiconformal $F_{n}$ is needed. The usual good convergence, see Lehto and Virtanen [15], p. 185, ensures $\partial F_{n} \rightarrow \partial F$ (a.e.).

Assume $\left\|\mu_{n}\right\|_{\infty},\|\mu\|_{\infty} \leq k \leq 1$ fixed. We assume that

(i) $F_{n} \rightarrow F$ uniformly on compact sets.

(ii) $\mu_{n}(z) \rightarrow \mu(z)$ (a.e.) on compact sets.

Lemma 2 (Lehto, Virtanen [15], p. 216). For $k<1$, there are $p=p(k)>2$ so that the above conditions imply $\left\|\partial F^{n}-\partial F\right\|_{p} \rightarrow 0$ on compact subsets of $\mathbf{R}^{2}$.

\section{Proof of Theorem 1 (part 1)}

We have to obtain a well defined $\log \partial F$ for $F=F^{\mu}$ and $\mu \in M_{0}$. First we assume $\mu$ smooth and obtain a uniform estimate.

Lemma 3. For canonical $F=F^{\mu}, \mu \in C_{0}^{\infty} \cap M_{0}$,

$$
\|\log \partial F\|_{*} \leq K\left(\|\mu\|_{\infty}\right) .
$$

For fixed $k,\|\mu\|_{\infty} \leq k \leq 1$ and $F=F^{\mu}$, consider the following one parameter family $F^{\lambda}$ defined for $|\lambda|<1 / k$. Let $F^{\lambda}$ be the canonical map satisfying

$$
\bar{\partial} F^{\lambda}=\lambda \mu \partial F^{\lambda} \text {. }
$$

Observe by the Ahlfors-Bers theory that $\left(F^{\lambda}\right),\left(F^{\lambda}\right)^{-1}$ are $C^{\infty}$ and the function

$$
f^{\lambda} \equiv \log \partial F^{\lambda}
$$


depends holomorphically on $\lambda$. Thus we may write

$$
f^{\lambda}=\lambda \alpha_{1}+\lambda^{2} \alpha_{2}+\cdots
$$

where each $\alpha_{j}(z) \in C^{\infty}$, and the series converges for $|\lambda|<1 / k$.

Note that without loss of generality we may assume $k \geq \frac{1}{2}$ (otherwise the bounds still apply for smaller dilatations). Choose a number $\varrho$ depending continuously on $k$ so that

$$
1<\varrho<\frac{1}{k}
$$

Let $\Gamma$ be the positively-oriented circle $\{|\lambda|=\varrho\}$. Now we apply Lemma 1 to $F^{\lambda}$ for $\lambda \in \Gamma$, noting that the dilatation $\lambda \mu$, satisfies $\|\lambda \mu\|_{\infty}=K(k)<1$. Thus for $\lambda \in \Gamma$

$$
\left\|\operatorname{Re} f^{\lambda}\right\|_{*} \leq K(k)
$$

However

$$
\operatorname{Re} f^{\lambda}=\frac{1}{2}\left(\lambda \alpha_{1}+\overline{\lambda \alpha_{1}}\right)+\frac{1}{2}\left(\lambda^{2} a_{2}+\bar{\lambda} 2 \bar{\alpha}_{2}\right)+\cdots
$$

and therefore

$$
\alpha_{j}=\frac{1}{2 \pi i} \int_{\Gamma}\left(\operatorname{Re} f^{\lambda}\right) 2 \frac{d \lambda}{\lambda^{j+1}}
$$

and consequently

$$
\left\|\alpha_{j}\right\|_{*} \leq \frac{K(k)}{\varrho^{j}}
$$

Thus, evaluating $f^{1}=\log \partial F$

$$
\|\log \partial F\|_{*}=\left\|\alpha_{1}+a_{2}+\cdots\right\|_{*} \leq \frac{K(k)}{\varrho-1} \leq K^{\prime}(k) .
$$

In order to define $\log (\partial F)$ for general $\mu$ we proceed as follows. For any fixed $k>\|\mu\|_{\infty}$ choose a sequence $\mu_{n} \in C_{0}^{\infty}$ so that the conditions (i), (ii) of Lemma 2 hold. Let $F$ be the canonical solutions of

$$
\bar{\partial} F_{n}=\mu \partial F_{n}
$$

and

$$
f_{n}=\log \left(\partial F_{n}\right) .
$$

Now we apply Lemma 3 and the weak compactness of the unit ball of BMO. Thus there is an $f \in \mathrm{BMO}$ so that some subsequence $f_{n_{k}} \rightarrow f$ weakly. However by Lemma 2

$$
\left|\partial F_{n_{k}}\right| \rightarrow|\partial F| \quad \text { (a.e). }
$$


In particular,

$$
\log \left|\partial F_{n_{k}}\right| \rightarrow \log |\partial F| \quad \text { (a.e). }
$$

Now Lemma 2 implies

$$
\int\left(\log \left|\partial F_{n}\right|\right) u d x d y \rightarrow \int(\log |\partial F|) u d x d y
$$

for all $u \in E$. Thus

$$
\operatorname{Re} f=\log |\partial F|
$$

and we may define

$$
\Pi(\mu)=f .
$$

Let us show $f$ is well defined. Now suppose $\nu_{n}$ is another sequence in $C_{0}^{\infty} \cap M$ converging to $\mu$ so that (i), (ii) hold. Let $g$ be the corresponding weak limit. For the sequence $\mu_{n}$ we associate an analytic family $\lambda \mu_{n},|\lambda|<1 / k^{\prime}, k^{\prime}>k$ and corresponding quasiconformal $F_{n}^{\lambda}$

$$
\partial F_{n}^{\lambda}=\lambda \mu_{n} \partial F_{n}^{\lambda}
$$

with

$$
f_{n}^{\lambda}=\log \left(\partial F_{n}^{\lambda}\right)
$$

which is a uniformly bounded holomorphic family in $\lambda$. Thus (at least for a subsequence) there is a weak limit $f^{\lambda}$, holomorphic in $\lambda$. By the above analysis

$$
\operatorname{Re} f^{\lambda}=\log \left|\partial F^{\lambda}\right|, \quad|\lambda|<\frac{1}{k^{\prime}} .
$$

Similarly for $\nu_{n}$ we define $\lambda \nu_{n}$ and corresponding $G_{n}^{\lambda}$

$$
\bar{\partial} G_{n}=\lambda \nu_{n} \partial G_{n}^{\lambda}
$$

with

$$
g_{n}^{\lambda}=\log \left(\partial G_{n}^{\lambda}\right) .
$$

Thus there is a weak limit $g^{\lambda}$ holomorphic in $|\lambda|<1 / k^{\prime}$. But as $\nu_{n} \rightarrow \mu$ a.e. $\lambda \nu_{n} \rightarrow \lambda \mu$ a.e. weakly and $\left|\partial G_{n}^{\lambda}\right| \rightarrow\left|\partial F^{\lambda}\right|$ (a.e). Therefore

$$
\operatorname{Re} g^{\lambda}=\log \left|\partial F^{\lambda}\right| \text {. }
$$

Now $g^{\lambda}, f^{\lambda}$ are holomorphic functions with the same real part and value (0) for $\lambda=0$. Thus $g^{\lambda} \equiv f^{\lambda}$ for $|\lambda|<1 / k^{\prime}$ and in particular (for $\lambda=1$ ) $g \equiv f$. 


\section{Proof of Theorem 1 (part 2)}

In this section we prove holomorphic dependence of $\log F^{\nu(\lambda)}$ for $\nu(\lambda)$ holomorphic in $\lambda$. By the Ahlfors-Bers theory $\log \partial F^{\nu(\lambda)}$ is holomorphic in $\lambda$ if $\nu(\lambda) \in C_{0}^{\infty} \cap M_{0}$.

For general holomorphic $\Lambda:\{|\lambda|<1\} \rightarrow M_{0}$ there exist sequences of holomorphic mappings $\Lambda_{n} \in C_{0}^{\infty} \cap M_{0}$ so that $\Lambda_{n}(\lambda) \rightarrow \Lambda(\lambda)$ a.e., in fact for any $r<\infty$

$$
\left\|\Lambda_{n}(\lambda)-\Lambda(\lambda)\right\|_{r} \rightarrow 0, \quad n \rightarrow \infty,
$$

and for $\lambda$ on a compact subset of the unit disk. To see this, take $\varphi_{n} \in C_{0}^{\infty}$ to be an approximation of the identity. Define

$$
\Lambda_{n}(\lambda)=\int \varphi_{n}(z-x-i y) \Lambda(\lambda) d x d y .
$$

Now as $\varphi_{n}>0, \int \varphi_{n} d x d y=1$,

$$
\left\|\Lambda_{n}(\lambda)\right\| \leq k<1 .
$$

Observe that $\Lambda_{n} \in M_{0} \cap C^{\infty}$ and that by the standard estimate, for all $r<\infty$,

$$
\left\|\Lambda_{n}(\lambda)-\Lambda(\lambda)\right\|_{r} \rightarrow 0
$$

for $\lambda$ on any (fixed) compact set of the unit disk.

Consequently the corresponding sequences

$$
g_{n}^{\lambda}=\log \left(\partial G_{n}^{\lambda}\right)
$$

will converge weakly to a holomorphic function $g^{\lambda}$. However, as before, this is the unique definition of $\log \left(\partial G^{\lambda}\right)$ which is therefore holomorphic in $\lambda$.

\section{Proof of Theorem 2}

As preliminary results we need the following form of the $\lambda$-lemma (see [7], [22]).

Lemma 5. Let $L$ be a holomorphic curve in $S$, i.e., $L=\Lambda(\{|\lambda|<1\})$,

$$
\Lambda:\{|\lambda|<1\} \rightarrow S
$$

is holomorphic with $\Lambda(0)=0$. Then for $|\lambda|<\frac{1}{3}$ there is a holomorphic map

$$
\Gamma:\left\{|\lambda|<\frac{1}{3}\right\} \rightarrow M(\Omega)
$$

so that

$$
\log \partial F(\lambda)=\Lambda(\lambda)
$$

This is because if $g=g^{\lambda} \in L$ depends holomorphically on $L$ then so does

$$
F^{\lambda}=\int^{z} e^{g^{\lambda}} d z
$$

which is univalent on $\Omega$. By the $\lambda$-lemma $F^{\lambda}$ extends to a quasiconformal mapping which, for $|\lambda|<\frac{1}{3}$, has dilatation $\mu^{\lambda}$ depending holomorphically on $\lambda$. 
Remark. The maximal complex manifold containing $g=0$ is essentially the class of all quasiconformal deformations with $\mu \in M(\Omega)$.

Since $\log \partial F^{\mu}=\log (1+\mathcal{H} \mu+\cdots), D \Pi(0)=\mathcal{H}$ and the above lemma gives

Lemma 6. The tangent space at 0 is

$$
V=\mathcal{H} L^{\infty}\left(\Omega^{C}\right)
$$

Remark. In particular there is a nontrivial complex manifold through 0 if and only if Area $\left(\Omega^{C}\right)>0$.

Finally we prove the theorem. Our previous comments give (i) $\rightarrow$ (ii) $\rightarrow$ (iii). We use Theorem 1 to complete the chain of equivalences. Now as $\mathcal{B}(\Omega)=$ $\mathcal{H} L^{\infty}\left(\Omega^{C}\right), \mathcal{H}$ is an open map of $L^{\infty}\left(\Omega^{C}\right)$ onto $\mathcal{B}(\Omega)$. However as $\left.\Pi\right|_{M(\Omega)}$ is holomorphic, by the implicit function theorem there is a $b>0$ so that $\Pi$ maps $\left\{\mu \in M(\Omega):\|\mu\|_{\infty}<b\right\}$ onto an open neighborhood of 0 . Thus $\Omega$ has univalence criteria.

\section{Proof of corollaries}

Observe that Corollaries 1, 2 are just special cases of Theorem 1. We next have to complete the proof of Corollary 3. For each $g \in \mathcal{B}(\Omega)$ we use the Cauchy integral to write

$$
g=g_{1}+g_{2}
$$

where $g_{j} \in \mathcal{B}\left(A_{j}^{C}\right)$. Thus if $\mathcal{B}\left(A_{j}^{C}\right)$ has integral representations so does $\mathcal{B}(\Omega)$. So by Theorem 2 we only have to prove the converse. Suppose now that $g \in \mathcal{B}\left(A_{1}^{C}\right)$ say. However, $g \in \mathcal{B}(\Omega)$ and there is a $t>0$ so that for $|\lambda|<t$

$$
\lambda g=\log F_{\lambda}^{\prime}
$$

for $F_{\lambda}$ univalent (normalized) on $\Omega$. But $g$ is analytic on $A$ so that $F_{\lambda}$ has an analytic extension to $A_{1}^{C}$. Also $F_{\lambda}$ is $1: 1$ on a set of Jordan curves $\gamma_{k}$ separating $A_{1}$ from $A_{2}$. Thus by the argument principle $F_{\lambda}$ is univalent inside $\gamma_{k}$. Consequently, $B\left(A_{1}^{C}\right)$ has univalence criteria.

Finally we prove Corollary 4 by simply observing that the second derivative of $\Pi$ with respect to $\mu$ must be in BMO:

$$
\log (\partial F)=\log (1+\mathcal{H} \mu+\mathcal{H} \mu \mathcal{H} \mu+\cdots)=\mathcal{H} \mu+\left(\mathcal{H} \mu \mathcal{H} \mu-\frac{1}{2}(\mathcal{H} \mu)^{2}\right)+\cdots
$$




\section{Proof of Theorem 3}

The method is almost exactly the same as the proof of Theorem 1 , so we only point out the differences. The first problem is defining $\log (\partial F)$ for $F, F^{-1} \in$ $C^{\infty}$. Now if $F$ has dilatation $\mu$, we use holomorphic family $F^{\lambda}$ defined by quasiconformal solutions of

$$
\bar{\partial} F^{\lambda}=\lambda \mu \partial F^{\lambda}, \quad|\lambda|<\frac{1}{\|\mu\|_{\infty}}
$$

fixing $0,1, \infty$. Now $F$ as holomorphically dependent on $\lambda$ we obtain a holomorphic function

$$
f^{\lambda}=\log \left(\partial F^{\lambda}\right)
$$

which letting $\log (1)=0$ defines $\log (\partial F)$. A homotopy argument shows the uniqueness of this definition. The rest of the proof proceeds analogously.

\section{VMO and quasiconformal mapping}

A function $f \in \mathrm{VMO}$ if

$$
f_{Q}\left|f-\int f d x d y\right| d x d y \rightarrow 0
$$

uniformly as area of all disks $Q$ satisfies $|Q| \rightarrow 0$. We consider the canonical map from the set of continuous dilatations to quasiconformal $F$ (fixing $0,1, \infty$ ).

Corollary 6. The canonical map

$$
\Pi(\mu)=\log \left(\partial F^{\mu}\right)
$$

is a well defined holomorphic map from $M \cap C$ into $\mathrm{VMO}_{\text {loc }}$. In particular for continuous $\mu, \log \operatorname{det}\left(D F^{\mu}\right) \in \mathrm{VMO}_{\text {loc }}$.

The proof is a simple deduction from Theorem 1. The canonical map is the restriction of a holomorphic map to a convex submanifold. We have only to show $\log (\partial F) \in \mathrm{VMO}_{\text {loc }}$. Now there exist $\mu_{n} \in M \cap C^{\infty}$ so that

$$
\left\|\mu-\mu_{n}\right\|_{\infty} \rightarrow 0 .
$$

Thus, by Theorem 3 , if $F=F^{\mu}$ and $F_{n}=F^{\mu_{n}}$

$$
\left\|\log \left(\partial F_{n}\right)-\log (\partial F)\right\|_{*} \rightarrow 0 .
$$

Thus $\log (\partial F)$ is in the BMO closure of $C$ and is therefore $\mathrm{VMO}_{\text {loc }}$.

Remark. One should note that for continuous $\mu \partial F$ is not necessarily even $L_{\mathrm{loc}}^{\infty}$. 


\section{References}

[1] Anderson, J.M., J. Clunie, and C. Pommerenke: On Bloch functions. - J. Reine Angew. Math. 270, 1974, 12-37.

[2] Ahlfors, L.: Quasiconformal reflections. - Acta Math. 109, 1963, 291-301.

[3] Ahlfors, L., and L. Bers: Riemann's mapping theorem for variable metrics. - Ann. of Math. 72, 1960, 385-404.

[4] Becker, J.: Über homöomorphe Fortsetzung schlichter Funktionen. - Ann. Acad. Sci. Fenn. Ser. A. I. Math. 538, 1973, 1-11.

[5] Bers, L.: A non-standard integral equation with applications to quasiconformal mappings. - Acta Math. 116, 1966, 112-134.

[6] Bers, L.: Oral communication. Conference on holomorphic functions, Berkeley, Mathematical Sciences Research Institute, 1986.

[7] Bers, L., and H. Royden: Holomorphic families of injections. - Acta Math. 157, 1986, 259-286.

[8] Colfman, R., R. Rochberg, and G. Weiss: Factorization theorems for Hardy spaces in several complex variables. - Ann. of Math. 109, 1976, 611-635.

[9] Fefferman, C., and E.M. Stein: $H^{p}$ spaces of several variables. - Acta Math. 129, 1961, 137-193.

[10] Genring, F.W.: The $L^{p}$ integrability of partial derivatives of quasiconformal mapping. - Acta Math. 130, 1973, 265-277.

[11] Gehring, F.W.: Univalent functions and the Schwarzian derivative. - Comm. Math. Helv. $52,1977,561-572$.

[12] Jones, P.W.: Extension theorems for BMO. - Indiana Math. J. 29, 1980, 41-65.

[13] IwANIEC, T.: The best constant in a BMO inequality for the Beurling-Ahlfors transform. - Michigan Math. J. no., 1987, 387-394.

[14] Kra, I.: Families of univalent functions and Kleinian groups. - Mathematical Sciences Research Institute, Berkeley, 1986.

[15] Lehto, O., and K.I. Virtanen: Quasiconformal mappings in the plane.- Springer-Verlag, Berlin-New York-Heidelberg, 1973.

[16] Nehari, Z.: The Schwarzian derivative and schlicht functions.- Bull. Amer. Math. Soc. $55,1949,545-551$.

[17] Osgood, B.: Univalence criteria in multiply connected domains. - Trans. Amer. Math. Soc. $260: 2,1980,459-473$.

[18] Reimann, H.M.: Functions of bounded mean oscillation and quasiconformal mappings. Comm. Math. Helv. 49, 1974, 260-276.

[19] Reimann, H.M.: On the parametric representation of quasiconformal mappings. - Symposia Math. XVIII, 1976, 421-428.

[20] Shiga, H.: Characterization of quasidisks and Teichmuller spaces. - Tôhuku Math. J. 37, $1985,541-552$.

[21] Strebel, K.: Ein Konvergensatz für Folgen quasikonfermer Abbildungen. - Comment. Math. Helv. 44, 1969, 469-475.

[22] Sullivan, D., and W. Thurston: Extending holomorphic motions. - Acta Math. 157, $1986,243-257$.

University of Maryland

Department of Mathematics

College Park, MD 20142

U.S.A.

Received 9 September 1987 